Radial and Nonradial Pulsations as Probes of Stellar Physics

ASP Conference Series, Vol. 259, 2002

C. Aerts, T.R. Bedding, 8 J. Christensen-Dalsgaard, eds.

\title{
Chromatic Amplitudes and Line-of-Sight Velocity Variations for the Pulsating DB White Dwarf GD 358
}

\section{R. Kotak}

Lund Observatory, Box 43, SE-22100, Lund, Sweden, e-mail: rubina@astro.lu.se

\begin{abstract}
We report on preliminary results from time-resolved optical spectroscopy of the He-rich variable white dwarf GD 358.
\end{abstract}

Similar driving mechanisms in white dwarfs with $\mathrm{H}$ and He-rich atmospheres are expected to give rise to similar trends in the pulsational properties across their respective instability strips (Brickhill, 1991; Wu, 2001). Figure 1 shows the Fourier Transforms of the brightness and line-of-sight velocity variations associated with the pulsations. We find significant velocity variations for most of the real modes. The ratio of velocity to flux variations provides an independent constraint on the spherical degree $(\ell)$ of the eigenmodes. The time-averaged spectrum (Fig. 2a) may be used to set limits on the presence of ${ }^{3} \mathrm{He}$ in the atmosphere; the inclusion of a ${ }^{3} \mathrm{He} /{ }^{4} \mathrm{He}$ transition zone in pulsational models has been shown to improve fits to the period spacings of the modes (Montgomery et al., 2001). Chromatic amplitudes can be used to infer the spherical degree of the modes and constrain the temperature stratification of the atmosphere. The chromatic amplitude for the strongest $(775 \mathrm{~s})$ mode is shown in Fig. $2 \mathrm{~b}$; note the different sensitivity of the lines to the pulsations. Its chromatic phase (Fig. 2c) shows signatures of velocity-induced variations; these are most evident in the line cores.

\section{References}

Brickhill, A.J. 1990, MNRAS, 246, 510

Montgomery, M.H., Metcalfe, T.S., \& Winget, D.E. 2001, ApJ, 548, L53

Wu, Y. 2001, MNRAS, 323, 248 


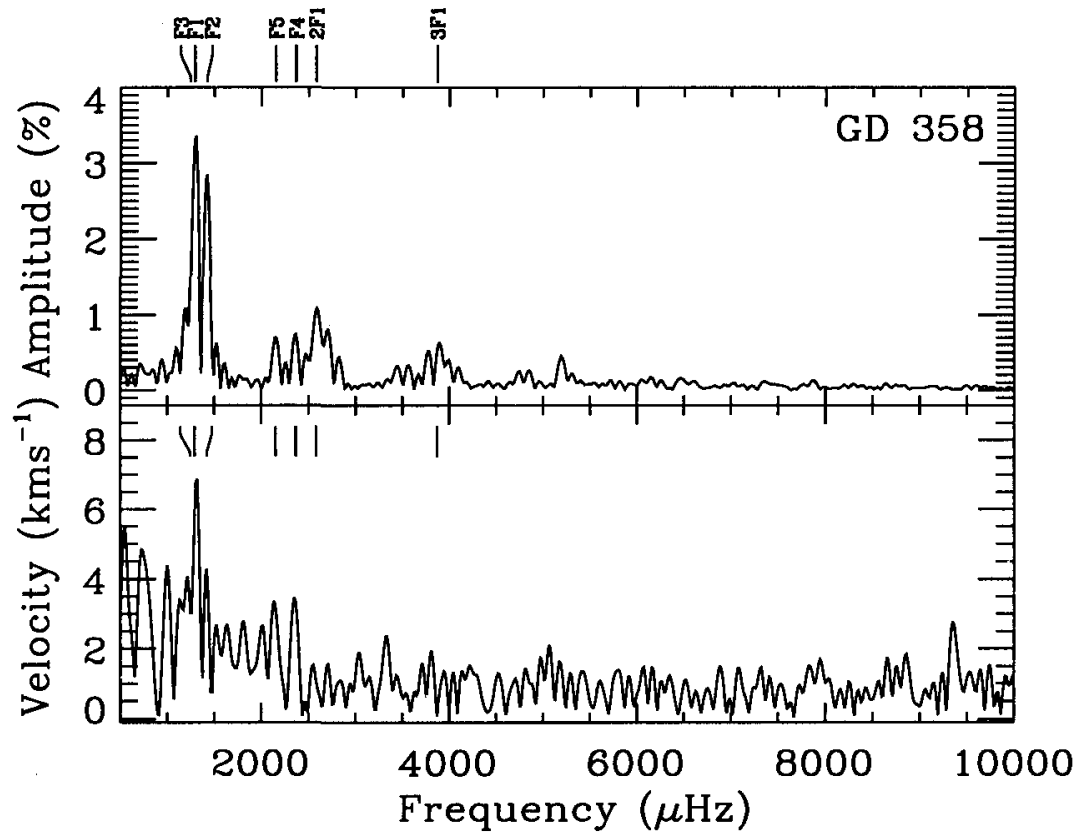

Figure 1. The Fourier Transforms of the light and velocity variations.

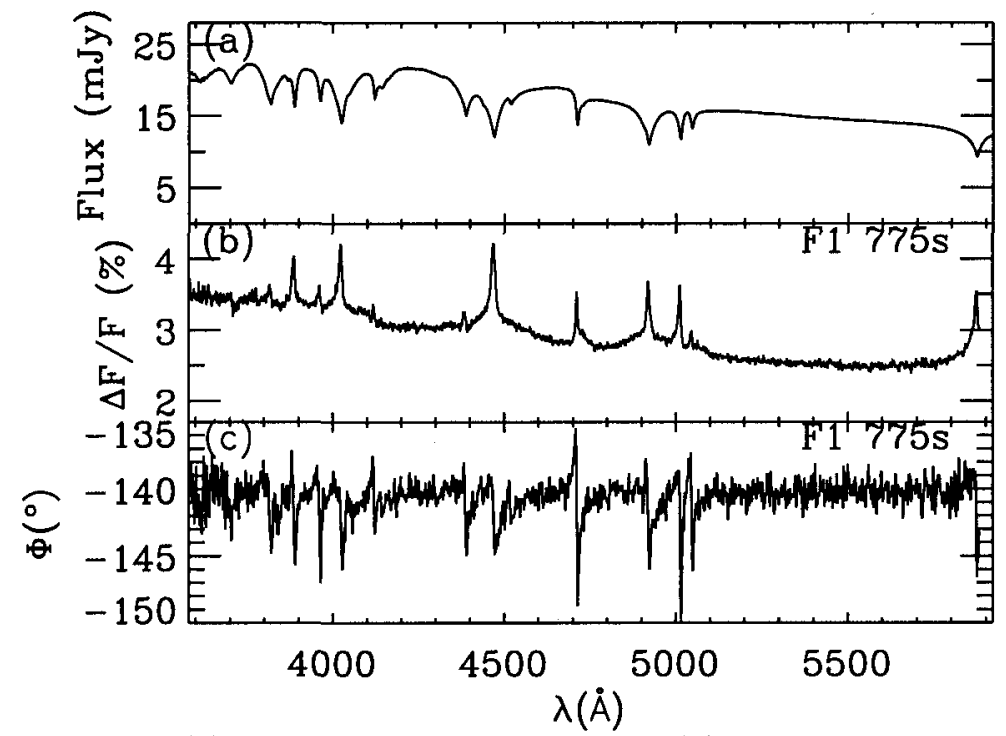

Figure 2. (a) the time-averaged spectrum; (b) chromatic amplitude for the strongest mode $(775 \mathrm{~s})$; (c) chromatic phase for the $775 \mathrm{~s}$ mode. 